\title{
Psicópatas versus trastornos de la personalidad: Matices clínico-conceptuales y aspectos diferenciales
}

\section{Psychopaths versus personality disorders: Clinical-conceptual nuances and differential aspects}

\author{
Juan Manuel Moreno-Manso, José Manuel Pozueco-Romero, Ma Elena García-Baamonde, \\ Macarena Blázquez-Alonso, Eloísa Guerrero-Barona, Mónica Guerrero-Molina. \\ Universidad de Extremadura, España \\ (Rec: julio de 2018 - Acept: mayo de 2019)
}

\section{Resumen}

La psicopatía ha sido frecuentemente relacionada con los trastornos de la personalidad, sobre todo con el subtipo antisocial, debido a las características delictivas que, en ocasiones, este implica. A pesar de esta asociación, y sin obviar los matices diferenciales, es importante recordar que los trastornos de la personalidad son diagnósticos clínicos, que vienen amparados por las nosologías psiquiátricas y recogidos en los manuales de trastornos mentales. En este sentido, no se debe entender la psicopatía como un trastorno mental, ni de la personalidad ni de cualquier otra índole psicopatológica. Si bien la psicopatía incorpora algunos rasgos aislados, también característicos de varios trastornos de la personalidad, es necesario establecer con exactitud los perfiles diferenciales entre estos y la psicopatía, ya que hay múltiples matices que podrán ayudar a establecer el diagnóstico diferencial pertinente y a evitar equiparar psicopatía con psicopatologías de la personalidad.

Palabras clave: Psicopatía; trastornos de la personalidad; psicopatologías; aspectos diferenciales.

\begin{abstract}
Psychopathy has been frequently associated with personality disorders, particularly with the antisocial subtype due to the criminal features that it sometimes entails. Despite this link, and without ignoring the differential nuances, it is important to remember that personality disorders are clinical diagnoses, which are supported by psychiatric nosology and included in manuals of mental disorders. Therefore, psychopathy should not be understood as a mental disorder, neither of personality nor of any other psychopathological nature. Even though psychopathy incorporates some isolated features, also characteristic of several personality disorders, it is necessary to accurately establish the differential profiles between them and psychopathy, since there are multiple nuances that may help to establish the necessary differential diagnosis and to avoid equating psychopathy with personality psychopathologies.
\end{abstract}

Key words: Psychopathy; personality disorders; differential aspects; psychopathologies 


\section{Introducción}

El fenómeno de la psicopatía no resulta sencillo de entender, y prueba de ello es que, en España, el estado de la cuestión sigue aún sin grandes cambios. Como en otros países, y a pesar de las pruebas empíricas contrarias a esta asunción generalizada, se le sigue conceptualizando como un tipo de trastorno de la personalidad (TP).

Por otro lado, es importante enfatizar que, tanto antaño como actualmente, muchos profesionales de la salud mental e investigadores equiparan, errónea y contantemente, psicopatía y trastorno antisocial de la personalidad (TAP). La constante relación entre los conceptos de psicopatía y TAP parece residir en otras dos de las numerosas y frecuentes asociaciones que se realizan; nos estamos refiriendo a la asociación entre psicopatía y conducta antisocial como la que se establece entre psicopatía y delincuencia.

A pesar de estas constantes y en ocasiones, erróneas asociaciones (dependiendo de las poblaciones de estudio), los matices diferenciales han sido reiteradamente subrayados en numerosos escritos. Por ejemplo, en cuanto a la relación entre psicopatía y trastornos mentales en general (ya sean trastornos de la personalidad o de cualquier otro tipo, según se recogen ampliamente en las nosologías psiquiátricas), esta polémica debería haber quedado ya disipada cuando Cleckley (1941/1976), entre los 16 rasgos característicos que delineó sobre la psicopatía, aclaró que uno de ellos es la completa ausencia de cualquier manifestación psicopatológica. Por otro lado, y en cuanto a la relación entre psicopatía y delincuencia, cabe señalar que se trata de otra polémica que realmente no existe, teniendo en cuenta dos puntualizaciones, una clínica y otra empírica: 1) que la conducta antisocial no es una característica definitoria de la psicopatía (Cleckley, 1976), y 2) que la conducta delictiva no es un componente central de la psicopatía (Skeem y Cooke, 2010).

Si bien las relaciones entre psicopatía y delincuencia sí han sido empírica y lógicamente demostradas en los estudios con psicópatas criminales, no ha sido el caso en los estudios con psicópatas integrados (o subclínicos, o no criminales). Sobre esta asociación, también hay que precisar una serie de matices de rigor, ya que ni la psicopatía es una condición necesaria para ser delincuente, ni la delincuencia es una característica exclusiva y necesaria para ser psicópata.

Finalmente, en este artículo también revisamos la puesta en tela de juicio de que los TP sean verdaderas enfermedades mentales. Autores tan renombrados como Theodore Millon son promotores de esta visión, existiendo disenso entre clínicos e investigadores; pese a ello, la American Psychiatric Association (APA) no ha hecho eco de ello en sus anteriores Diagnostic and Statistical Manual of Mental Disorders (DSM) ni tampoco en su actual DSM-5 (APA, 2013).

Ante tanta polémica y mirando el concreto perfil del psicópata, podemos comprobar que ninguna de sus características denota manifestaciones de sintomatología psicopatológica o de trastornos mentales. De igual forma, los aspectos diferenciales entre la psicopatía y los TP nos ayudarán a disipar estas dudas, de modo que así, el hecho de equiparar y/o confundir diagnósticos psicopatológicos o trastornos mentales con el diagnóstico de psicopatía, parecerá ser más una inclinación personal de cada autor que procedente de las demostraciones empíricas al respecto.

\section{Los trastornos de la personalidad (TP) y la psicopatía: matices clínico-conceptuales y aspectos candentes}

\section{Los TP: ¿Realmente son verdaderas enfermedades mentales?}

Se ha comentado que los TP son «un modo de ser y comportarse» (Belloch y Fernández-Álvarez, 2002, p. 12), pero esta asunción general y que, en cierto modo, da pie a mirar los TP desde un punto de vista dimensional, precisa matices. Si bien actualmente la APA (2013) ha suprimido el tradicional sistema multiaxial (Sandín, 2013), no hay que olvidar que el punto de vista categorial en el que se siguen basando los diversos diagnósticos psicopatológicos viene influenciado por dicho sistema multiaxial, en el cual el Eje II estaba -en parte- dedicado a los TP. Y es en este sentido en el que se afirma que: «a partir de ese momento, toma progresivamente fuerza la necesidad de una evaluación multiaxial, que considera la personalidad en la que se muestran o manifiestan los síndromes clínicos» (Ortíz-Tallo, Cardenal, Ferragut y Cerezo, 2011, p. 50).

Por otro lado, también es importante conocer que el abordaje de los pacientes con TP, está mediatizado por la dificultad que tienen para formarse una representación de los demás que sea razonable, polifacética y realista (incluidos sus terapeutas), repercutiendo en sus relaciones interpersonales (Belloch, 2010). En esta misma línea, otros autores han especificado que los pacientes con TP: "...tienen problemas para comprender que no son siempre el centro principal de los pensamientos de los demás o que estos pueden tener una perspectiva diferente de la suya. Dicha dificultad en descentrarse de sus propios estados mentales y emocionales está en el corazón de las disfunciones de la personalidad" (Prado, García y Sánchez, 2013, p. 139).

Precisamente sobre este núcleo psicopatológico existe un importante consenso en la literatura, que señala que los TP se componen de disfunciones del self (o sí-mismo) y de patrones interpersonales inflexibles y crónicos (Bateman y Fonagy, 2004; Clarkin, 2006). Como subraya Belloch (2010), se trata de alteraciones del funcionamiento global de la persona, de su modo de ser y de estar en el mundo, lo que los diferencia de los síndromes clínicos o trastornos mentales del Eje I del DSM, de naturaleza más circunscrita. Sin embargo, como han puesto de manifiesto otros autores, «la formulación de los trastornos de personalidad en psicopatología se ha hecho al margen del desarrollo de la psicología de la personalidad» (Pelechano, De Miguel y Hernández, 2009, p. 477), y este proceder, lógicamente, ha tenido sus consecuencias en forma de críticas y detractores del modelo psicopatológico de la personalidad.

Así, en este sentido, es importante recordar que hay autores especialistas en materia de personalidad que incluso han llegado a asegurar que los TP no son verdaderas enfermedades mentales (Millon, 1981). Son repetidas las veces que muchos clínicos no reparan en establecer los necesarios matices diferenciales existentes entre lo normal, lo anormal y lo (psico)patológico dentro del complejo tópico de la personalidad. Según el doctor Theodore Millon y sus colegas, hay 3 características diferenciales entre las personalidades normales y los TP (MiIlon, 1981, 2011; Millon y Davis, 1995): 
a) La capacidad de funcionar de un modo autónomo y competente.

b) La posibilidad de ajustarse de una manera eficiente y flexible al medio social.

c) La capacidad de conseguir las metas propias, con el subsiguiente sentimiento de satisfacción subjetiva.

Vistos de este modo, y en notable contraste con la perspectiva psicopatológica imperante tanto de la APA y como de la Organización Mundial de la Salud (OMS), defendida en sus respectivos manuales de trastornos mentales o psicopatologías, otros muchos autores desvinculados de este enfoque y que adoptan una perspectiva psicológica han vuelto a recordar que los TP aparecen en personas psíquicamente normales, que razonan bien y con un sentido claro de la realidad. Por tanto, sostienen que los TP no son propiamente enfermedades mentales, como sí están convencidos de que sí lo son las neurosis y las psicosis (Millon, Grossman, Millon, Meagher y Ramnath, 2006).

\section{Las personalidades psicopáticas: Antecedentes para la confusión psicopatológica}

Además de los matices realizados sobre los TP en el apartado anterior, hay algunos más que resultan necesarios de hacer para entender, posteriormente, tanto las características aparentemente compartidas entre los TP y la psicopatía, así como también sus diferencias esenciales. Para ello recurriremos, en parte, al capítulo "Personalidad y Trastorno de la Personalidad", incluido en el libro Tratado de Trastornos de la Personalidad, de la profesora Amparo Belloch (2010), donde plantea una cuestión como la siguiente: "¿trastorno de la personalidad o personalidades trastornadas?" Frente a esta cuestión, la misma autora responde ampliamente a través de una serie de precisiones y conceptos clarificadores, que creemos ayudarán a lo que pretendemos establecer aquí: las características comunes y diferenciales entre los TP, en general, y la psicopatía, en particular. No obstante, antes de eso tenemos que realizar una especie de regreso al pasado, a ciertos momentos e hitos de la historia de la psicopatología y de la psicopatía para entender el problema de fondo que subyace a la actual confusión y equiparación terminológicas, que aún permanece vigente.

Teniendo en cuenta que la psicopatía es un término que tiene ya más de 200 años de historia (Millon, Simonsen y Birket-Smith, 1998), esta se ha caracterizado, en un primer momento, por una perspectiva moralista, encabezada por autores como Pinel (con su concepto de manía sin delirio), Rush (depravación moral innata) y Prichard (locura moral) y, en un segundo momento, por una perspectiva biológico-atavista y degeneracionista-hereditarista, encabezada por autores y sus conceptos como Morel (locura de los degenerados), Koch (inferioridad psicopática congénita) y Lombroso (criminal nato/atávico). Tras estas perspectivas, comenzaron a aparecer autores a los que podríamos englobar dentro de la denominada perspectiva psicopatológica, quienes aclararon algunos aspectos controvertidos sobre la psicopatía, pero también contribuyeron a generar más confusión respecto a este término. Entre estos autores destacan, de manera notable, dos: Emil Kraepelin y Kurt Schneider.

En un principio, y esto conviene recordarlo, "Kraepelin consideró los TP como formas intermedias de psicosis, defen- diendo la idea de continuidad entre esta y aquellos" (Belloch y Fernández-Álvarez, 2002, p. 14). Como era de esperar, la terminología de inferioridad psicopática que propuso Koch en 1891 fue bien aceptada por los psiquiatras de la época, siendo recogida por el conocido psiquiatra alemán Emil Kraepelin. En 1896, Kraepelin propuso el concepto de estados psicopáticos que posteriormente, en su obra de 1904, culminó con el concepto más conocido de psychopathischen persönlichkeiten (personalidades psicopáticas), denominando así a ciertas alteraciones congénitas de la personalidad caracterizadas por una anormalidad permanente del área afectiva-conativa, diferenciándolas de los citados originären krankheitszustände (estados psicopáticos), que seguían el curso de un trastorno mental.

Kraepelin propuso el término que hoy en día utilizamos de personalidad psicopática, para referirse a aquellas "personalidades pobremente dotadas por influencias hereditarias, dañadas en sus procesos germinativos y otras influencias físicas tempranas en el desarrollo" (citado en Millon et al., 1998, p. 9), cuya condición forma parte de un proceso irreversible, idea que desgraciadamente perdura hasta nuestros días. Tras una serie de modificaciones de sus primeros postulados, en 1909/1915 Kraepelin define a los psicópatas como "personas con déficit de los afectos o de la voluntad" (citado en Millon y Davis, 1998, p. 451), a los cuales dividió en dos grupos: por un lado, sujetos que poseían una disposición mórbida, obsesiva, impulsiva y desviada sexualmente, y por otro lado, sujetos que manifestaban peculiaridades en su personalidad, dentro de los que distinguió a los excitables, los inestables, los impulsivos, los mentirosos y timadores, los pendencieros y los antisociales. En relación a estos últimos, Kraepelin, en su obra de 1909/1915, sienta los precedentes de lo que sería la nomenclatura actual, caracterizándolos como:

"(...) los enemigos de la sociedad... caracterizados por una falta de elementos morales. Suelen ser destructivos y amenazadores (...), carecen de una reactividad emocional profunda; poseen poca capacidad de comprensión y afecto. Es muy probable que hayan tenido problemas en la escuela, siendo proclives al absentismo. Suelen empezar a robar desde muy jóvenes y cometen crímenes de varios tipos" (citado en Partridge, 1930, pp. 88-89).

Sin duda alguna, las tesis de Kraepelin van a notar su influjo en autores posteriores, algunos incluso discípulos suyos. Aunque el intento de este autor por separar a las personalidades psicopáticas de otros trastornos mentales fue encomiable, es cierto que su postura era aún ostensiblemente de corte psicopatológica, por lo que no desdeñaba la posibilidad de que la personalidad anormal de aquellos "psicópatas" que él estudió estuviera, en algún momento, a caballo entre las psicosis y las neurosis, y mucho más cerca de aquellas que de estas. De esta manera, y a diferencia de lo que se sostiene hoy, los psicópatas podrían enfermar mentalmente en algún momento. Posteriormente, uno de los discípulos de Kraepelin, el también psiquiatra alemán Kurt Schneider (1923), describió a las personalidades psicopáticas como aquellas personalidades anormales que sufren por su anormalidad, o cuya anormalidad causa sufrimiento a la sociedad. Destacó que este tipo de individuos se caracterizan por una vida emocional poco intensa, y carecen de vergüenza, decencia, remordimiento y conciencia. También señaló que estos individuos son descorteses, fríos e irritables, y muy violentos cuando cometen crímenes; cono- 
cen y entienden el código moral social, pero son indiferentes hacia él. Además, este autor propuso la existencia de diez subtipos de "psicópatas", caracterizando y designando a cada uno de ellos según sus rasgos más prominentes. Resulta, en este sentido, ostensible el paralelismo existente entre estos diez subtipos de "psicópatas" y los diez tipos de TP de la APA. Aunque reforzó la idea de que muchos de estos individuos eran delincuentes desde jóvenes e incorregibles, Schneider creía que además de estos sujetos que terminaban cometiendo actos delictuales, existían algunos viviendo dentro de la sociedad en forma normal y muchos de ellos presentaban un éxito inusual en el ámbito político o económico. Con su obra principal Dies Psychopathischen Personlichkeiten (Las Personalidades Psicopáticas), Schneider (1923, 1980) anticipó ciertos problemas contemporáneos relacionados con estos "psicópatas", refiriéndose a las ideas de los especialistas que le habían precedido muchas décadas antes y se habían enfrentado a los mismos problemas. Escribió lo siguiente:

"(...) que el término locura moral era probablemente el más utilizado en la medicina forense y urgía a todas las personas que trabajaban en el mundo legal a tener cuidado para que el pretexto de la locura moral no les arrebatase de las manos la espada de la justicia. No nos parece apropiado en los casos legales promover el pretexto de una responsabilidad disminuida en estas personalidades solamente porque puede ser la única forma de ingresarlos en una institución. Nos parece una cuestión de principios que los juicios sobre la culpabilidad no residan únicamente en consideraciones legales" (Schneider, 1923, pp. 131 y 132).

De este modo, Schneider entendía la psicopatía de cuatro maneras: como un modelo de constitución hereditaria y estática, como una anomalía del carácter, como un tipo asocial, y como una estructura morbosa endotímica vital.

Una vez más, como vemos, la historia del concepto de psicopatía nos revela la polémica existente y la dificultad que tenían esos diversos autores para intentar conceptualizar a los psicópatas fuera de una perspectiva psicopatológica. ¡Qué duda cabe que todos esos antiguos vocablos le hicieron un flaco favor al concepto de psicopatía para la posteridad! Los franceses estaban empecinados con el término manie sans délire, los ingleses estaban obsesionados con el de moral insanity y los alemanes vinieron a empeorar aún más el estado de la cuestión cuando Schneider (1923), en su libro, estableció una clasificación de hasta 10 tipos de personalidades psicopáticas, en las que aglutinaba cualquier forma de psicopatología. Así, habló de psicópatas lábiles de ánimo, depresivos, hipertímicos, inseguros de sí mismos, necesitados de estimación, fanáticos, explosivos, desalmados, abúlicos y asténicos.

Sin duda alguna, lo que hizo Schneider (1923) no fue otra cosa que llamar personalidades psicopáticas a todas aqueIlas personalidades anormales que actualmente se conciben en la psiquiatría como trastornos de la personalidad. Así, por ejemplo, las psicopatías explosivas de Schneider se denominan ahora (según la APA) trastorno límite de la personalidad, trastorno histriónico de la personalidad e incluso trastorno explosivo-intermitente. En suma, para este autor alemán, todas las enfermedades mentales actuales eran personalidades psicopáticas. Probablemente, el mayor acierto que quizás tuvo Schneider respecto al moderno concepto de psicopatía, fue cuando señaló que los psicópatas no solo se hallan en las pri- siones e instituciones psiquiátricas, sino en toda la sociedad, ya que muchas veces eran personas que tenían éxito en los negocios y en la vida social mundana, ostentando incluso posiciones de poder en la política, en las empresas y en otros diversos ámbitos.

\section{Las personalidades antisociales y los delincuentes: más antecedentes para la constante equiparación psicopato- lógica y delictiva}

Pero la confusión terminológica se agravó aún más cuando la APA, en sus sucesivos DSM, vino empleando el amplio y abstracto concepto de personalidades antisociales, concepto que al final cristalizó en el actual de TAP. Más grave aún para la confusión diagnóstica, fue la equiparación terminológica que propuso la misma APA, concretamente en su DSM-IV, al sugerir que se emplearan de manera intercambiable todos los conceptos relacionados con la psicopatía (como si fueran sinónimos), al aseverar textualmente que el patrón conductual definitorio del TAP "también se ha denominado psicopatía, sociopatía o trastorno disocial de la personalidad" (APA, 1994, p. 655).

El término psicopatía ha sido mal utilizado y mal interpretado, por haberlo equiparado de manera constante y casi en exclusiva con el término más amplio de personalidades antisociales (Lykken, 1995). Pese a que el TAP (o trastorno disocial de la personalidad según la OMS) y la psicopatía comparten algunas características (específica y fundamentalmente algunos rasgos relativos a la vertiente conductual), ambos conceptos aluden a constructos diferentes (Lykken, 1995; Millon et al., 2006). Además, hay que precisar que los indicadores de desviación conductual son incapaces de distinguir la psicopatía de otras condiciones frecuentemente relacionadas con conducta antisocial y/o criminal (Lykken, 1995).

Por otro lado, como ya anticipamos, la otra constante equiparación estriba en la casi exclusiva relación entre psicopatía y delincuencia que numerosos estudios han realizado, asociaciones que, lógicamente y en algunos casos, solo pueden realizarse en esos estudios que emplean muestras penitenciarias. Desde luego que "la psicopatía es uno de los constructos que permiten comprender cómo un sujeto puede elicitar una conducta transgresora de las normas sociales" (León y Zúñiga, 2012, p. 1198), pero el significado de "conducta transgresora de las normas sociales" no siempre ni solo se circunscribe a conductas antisociales. Así, por ejemplo, la mayoría percibimos como una "norma social" no escrita y aceptada el hecho de no llevar abrigo y bufanda en verano, pero: ¿sería antisocial llevar ambas prendas en pleno verano, o simplemente sería atípico, poco frecuente? Aunque sobre este particular no podemos dedicar aquí más espacio, sí que debemos volver a poner de relieve una serie de matices fundamentales tanto sobre la versión de la personalidad y conducta antisociales de la psicopatía como de la versión delictiva de la misma. En este sentido, tales matices los podemos resumir tal como lo recogen algunos autores en sus investigaciones y en los siguientes términos:

"A diferencia del trastorno disocial, la psicopatía no pivota únicamente en torno al comportamiento, sino a dimensiones entre las que sobresalen el narcisismo, insensibilidad emocional e impulsividad, que se toman como predictoras de los problemas de conducta. (...) Asimismo, la psicopatía no es equipa- 
rable a la delincuencia legal, ya que no todos los delincuentes son psicópatas, ni todos los psicópatas son delincuentes (Fariña, Vázquez y Arce, 2014, p. 882).

\section{Psicopatía versus TP: Aspectos diferenciales}

El perfil general del psicópata: Características de personalidad, emociones y conductas

Cuando estudiamos los factores cognitivo-conductuales de la psicopatía, encontramos en la estructura de personalidad y emociones centrales del psicópata, un importante rasgo de alto egocentrismo-narcisismo (Cleckley, 1976; Hare, 1993, 2003a; Millon, 1981). Podría decirse que una de las claves definitorias de estos sujetos es su extremado sentido de autovaloración grandilocuente, es decir, una exaltación extremada de la propia personalidad, hasta considerarla como centro de la atención y actividad generales. Estos individuos intentarán demostrar su estatus y cualidades superiores pisoteando y humillando a los demás.

Tratamos con un ser egoísta y, en muchas ocasiones y en algunos casos, irresponsable e indiferente ante las normas. Muchos de estos individuos tienden a vivir al día y no les preocupa el futuro, mientras que otros individuos psicópatas parecen estar mejor integrados en la sociedad y son más controlados en muchos aspectos de su vida, dando una completa apariencia de normalidad (Pozueco, 2010). Cuando los psicópatas son más impulsivos que controlados, y debido a su necesidad inmediata de estimulación y la constante búsqueda de nuevas sensaciones que les hagan sentirse vivos o ser alguien en el mundo, las metas o fines que buscan suelen ser a corto plazo. Así, sus intereses se focalizan en el ambiente inmediato y perciben a los demás como objetos o cosas (cosificación), que manipulan para conseguir sus objetivos. En efecto, las personas son vistas como un objeto cualquiera y serán percibas por el psicópata como un obstáculo ambiental que interfiere en sus deseos. En algunos casos, estos individuos se caracterizan por un estilo de vida inestable, parásito y sin propósito, dependen de los demás y no intentan conseguir una estabilidad e independencia en sus vidas, mientras que, en casos de psicópatas más integrados, socializados y con éxito, sus vidas están más que resueltas, yendo sus anhelos más allá de parasitar económicamente en los demás. Asimismo, sus objetivos responden a intenciones o propósitos para los que emplean tácticas de engaño y manipulación mucho más elaboradas.

Para obtener los fines anhelados, el psicópata se provee de un gran encanto superficial (Hare, 1993), algo que es favorecido por tener, en algunos casos, una personalidad extrovertida (Eysenck, 1977), mientras que otros psicópatas, con un estilo de vida más controlado y nada impulsivo, suelen más introvertidos (Pozueco, 2010). En general, se trata de un individuo locuaz y encantador, de una persona amena, entretenida, divertida y muy ingeniosa, que suele dar una buena imagen de sí misma. De igual manera, se muestra como un individuo muy seguro y no se considera nunca como un fracasado. La mayor parte de sus acciones suele venir acompañada de mentiras que, por lo general, son reiterativas y que incluso casi llega a creer el mismo, mentiras a las cuales incluso suele "darles la vuelta" para hacerlas nuevamente creíbles si percibe que su interlocutor se ha dado cuenta de que le miente (Pozueco, 2010). Para estos sujetos, la mentira y el engaño son parte integradora de su relación con los demás (Cleckley, 1976). Asi- mismo, utilizarán la mentira y el engaño para estafar, engañar y manipular a otros (Hare, 2003a). Debemos recordar que el psicópata es un gran manipulador, que se sirve de cualquiera en su propio beneficio personal.

Podemos decir del psicópata que se trata de un actor consumado (Hare, 1970); de hecho, en sus relaciones con los demás puede demostrar un afecto superficial pero poco duradero, siendo incapaz de describir estados afectivos más profundos. En este sentido, se dice que el psicópata mima e iamita las emociones humanas, pero sin sentirlas de manera genuina (Cleckley, 1976), lo que más gráficamente expresaron otros autores con la frase: "el psicópata se sabe la letra, pero no la música" de las emociones (Johns y Quay, 1962, p. 217). Aunque se vean -en algunos casos y en algunos momentos- rodeados de muchas personas (no olvidemos su notable encanto superficial), el psicópata, en general, es un individuo solitario: no suele necesitar a los demás a menos que perciba que de los mismos va a obtener beneficios personales.

En algunos casos -pero no en todos-, y si tenemos en cuenta que otra característica típica de la psicopatía es su insinceridad y falsedad, nos encontramos con psicópatas que muestran una ausencia de relaciones personales genuinas y duraderas, así como la preferencia por relaciones sexuales triviales, impersonales y poco integradas (Cleckley, 1976). Todas estas características que venimos perfilando hasta aquí, son medios que utilizan los psicópatas para conseguir sus fines, lo que les confiere rasgos propios de la personalidad maquiavélica (Pozueco, 2010; Pozueco, Moreno, Blázquez y García-Baamonde, 2013).

Además de todas esas características, el rasgo más prominente y sin duda, central de la psicopatía, es la ausencia de empatía, que desemboca en otro rasgo central y típico de los psicópatas: la ausencia de remordimientos y/o de sentimientos de culpa (Cleckley, 1976; Hare, 1993, 2003a; McCord y McCord, 1964). Así, estos individuos muestran una ausencia general de consideración por las consecuencias de sus acciones y no sienten remordimientos por los efectos producidos en sus víctimas. Su falta de empatía es manifiesta: son incapaces de ponerse en el lugar de los demás, con lo cual demuestra una fuerte insensibilidad y crueldad (Hare, 2003a). En algunas ocasiones su actuación será más bien impulsiva por esa necesidad inmediata de estímulos (Hare, 1982), mientras que en otras se conducen con extremada cautela y controlando sus impulsos para no dejar entrever sus verdaderas intenciones (Hare, 1993; Pozueco, 2010), pudiendo llegar a mostrar una gran frialdad en actos muy meditados y elaborados (Wells, 1988).

En definitiva, y analizando el perfil general que acabamos de exponer, algunas características de la psicopatía relativas a conductas antisociales y delictivas nos hacen pensar, lógicamente, que este tipo de individuos tienen más probabilidades de entrar con frecuencia en contacto con el sistema judicial los psicópatas criminales-, mientras que otros que no incurren en este tipo de conductas -los psicópatas subclínicos- están aparentemente mejor integrados entre nosotros y nos pueden arruinar la vida y colarse en numerosos ámbitos y de múltiples maneras que no necesariamente implican conductas delictivas. Así, ninguna de las características de la psicopatía podría llevar a pensar que estos individuos tengan contactos con instituciones psiquiátricas, a menos que se defienda una 
perspectiva psicopatológica y que, en alguno que otro informe pericial, se argumente que la psicopatía es un tipo de trastorno mental que, como tal, conlleva eximentes y/o atenuantes de la responsabilidad criminal o imputabilidad, aspectos que en absoluto son ciertos y que ya han sido reiteradamente puestos de relieve (Malatesti y McMillan, 2010; Pozueco, 2011).

\section{La psicopatía y los TP en general: características comu- nes y diferencias esenciales}

Como decíamos al principio de este artículo, a pesar de las pruebas empíricas contrarias a la asunción generalizada, tanto en España como en otros muchos países se sigue conceptualizando a la psicopatía como un tipo de TP, olvidando aspectos tan importantes como que la psicopatía se caracteriza por una ausencia absoluta de cualquier manifestación de psicopatología (Cleckley, 1976). Si bien hay ciertas semejanzas entre algunas características aisladas y dispersas de los diversos TP y algunas características de la psicopatía, desde luego las diferencias son mayores.

Entre las características comunes entre la psicopatía y los TP, la que sin duda destaca por encima de cualquier otra, la podemos encontrar en las siguientes apreciaciones que realiza Belloch (2010) con respecto a los TP:

"Los TP se caracterizan por su aparición temprana en la vida del individuo, su estabilidad, y su resistencia al cambio. Recuérdese que uno de los elementos clave de la personalidad es el hecho de que permanece relativamente estable a lo largo de toda la vida. $Y$ esto se aplica tanto a las personalidades normales, como a las trastornadas (Belloch, 2010, pp. 21-22)".

Sin equiparar de ninguna manera psicopatía y TP, parece ser que algunos autores están de acuerdo en admitir que los primeros síntomas o rasgos psicopáticos comienzan a manifestarse ya en una edad muy temprana de la vida, incluso a partir de los tres años (Hare, 1993). Por otro lado, como la psicopatía también conlleva rasgos de personalidad, es lógico pensar que los individuos que la perfilan los mantienen relativamente estables a lo largo de toda su vida; de hecho, la psicopatía no se pasa -no remite- con la edad: si bien ciertas conductas antisociales y delictivas típicas de los psicópatas criminales tienden a desparecer al mismo tiempo que su vigor físico, los rasgos característicos de su personalidad y emociones permanecen prácticamente inalterables durante toda su vida (Hare, 1993). Esta asunción es lógica si nos atenemos al hecho que, por ejemplo, para engañar y manipular a los demás no es necesario incurrir en actividades delictivas, como tampoco lo es para primero seducir a alguien y luego parasitarlo hasta límites insospechados, pero no delictivos, y todo ello sin ningún tipo de escrúpulos ni remordimientos (Pozueco, 2010).

Otra de las pocas características que la psicopatía puede compartir con los TP es la referente a que los sujetos con TP "tienen problemas para comprender que no son siempre el centro principal de los pensamientos de los demás o que estos pueden tener una perspectiva diferente de la suya" (Prado et al., 2013, p. 139). En este sentido, el encanto superficial y el sentido grandilocuente de autovalía típicos de los psicópatas (Cleckley, 1976; Hare, 1993), son rasgos que suelen ponerlos en el centro de atención de numerosas situaciones, ya que es fácil verlos querer destacar por encima de los demás, sin tener en cuenta otras perspectivas y sin el menor reparo, lo que no les ocasiona ningún tipo de disfunción en su estructura de personalidad.

Sin embargo, las diferencias entre los TP y la psicopatía son más numerosas y evidentes que sus aparentes similitudes. Si tenemos en cuenta que los TP son trastornos mentales según los concibe la APA, también es preciso recordar que "los trastornos mentales producen un sufrimiento personal importante, que impregna muchos aspectos de la experiencia de la persona y modifica su forma de "estar en el mundo" y de experimentarlo (en muchos casos, de manera dramática)" (Belloch, 2010, p. 22). Los psicópatas, tal como actualmente se conciben, no tienen sufrimientos personales ni por su forma de ser ni mucho menos por su forma de actuar, ni por las negativas consecuencias que generan en los demás (Garrido, 2000; Hare, 1993). En efecto, llegan al punto de ser incapaces de ponerse en el lugar de los demás para ver los agravios que les ocasionan, y todo ello sin el menor atisbo de culpa ni arrepentimiento (Cleckley, 1976).

Según Belloch (2010, p. 23), cuando hablamos de que un individuo tiene un TP nos referimos a que su modo de ser habitual es enfermizo, patológico, anormal o disfuncional, ya sea porque no es el modo de ser más frecuente de las personas de su entorno, porque no se ajusta a lo que cabría esperar de él teniendo en cuenta su contexto socio-cultural, su formación, los roles que se supone debería asumir, etc., o porque no le permite desarrollar sus capacidades potenciales de una forma positiva y adecuada. Sin embargo, estas premisas son difícil de trasladar al caso de los psicópatas, sobre todo al caso de los psicópatas integrados, y ello por varias razones:

a) Porque la psicopatía no contiene ni un solo rasgo que haga pensar que tal individuo psicopático tenga un modo de ser enfermizo o patológico (Cleckley, 1976).

b) Porque es difícil comprobar si todas las personas de su entorno también son como él/ella y si se comportan "así" de manera frecuente, sobre todo si tenemos en cuenta que la impostura y los impostores abundan entre los psicópatas integrados (Garrido, 2004), además de tener en cuenta que algunas personas son psicópatas y otras solamente lo parecen (Marietán, 2011).

c) Porque es difícil entender que, por ejemplo, personas bien posicionadas y reconocidas no se ajustan al perfil psicopático precisamente por esas posiciones que ostentan, las que también pueden servir de "tapadera" o "doble fachada" (Garrido, 2000).

d) Porque la mayoría de los psicópatas integrados -aunque comentan un delito y pasen a la categoría de psicópatas criminales- tienen habilidades y capacidades destacadas, que les permiten lograra a menudo cualquier cosa que se marquen como objetivo (Hare, 1993). Cabe señalar, que lo anterior es independiente de si su objetivo tiene un carácter positivo y adecuado o negativo e inadecuado, y que en el complejo mundo en el que vivimos, es realmente difícil dirimir en qué fase del ciclo de manipulación psicopático podrían encontrarse ciertas personas (Pozueco, 2010)-.

\section{Discusión}

Los trastornos mentales han sido descritos como patrones de comportamiento con significación clínica que aparecen 
asociados a un malestar emocional o físico de la persona, a una discapacidad, al deterioro del funcionamiento cotidiano, a la pérdida de libertad o incluso a un riesgo de implicarse en conductas contraproducentes o de morir prematuramente (Echeburúa, Salaberría y Cruz, 2014).

Por su parte los TP, desde una perspectiva categorial (unidimensional, unitaria, taxativa y procedente de la psiquiatría), son definidos como un patrón permanente e inflexible de experiencia interna y de comportamiento que se aparta acusadamente de las expectativas de la cultura del sujeto, tiene su inicio en la adolescencia o principio de la edad adulta, es estable a lo largo del tiempo y comporta malestar o perjuicios para el sujeto (APA, 2013, p. 645). Además, los TP tienen las siguientes características básicas: a) están profundamente enraizados y son de naturaleza inflexible, b) son desadaptativos, especialmente en contextos interpersonales, c) son relativamente estables a lo largo del tiempo, d) deterioran de forma significativa la capacidad de la persona para funcionar, y e) producen malestar en el entorno de la persona (Caballo, Salazar e Irurtia, 2014).

Sin embargo, desde una perspectiva dimensional (más heterogénea, ni taxativa ni categórica, y procedente de un paradigma psicosocial), se hace realmente difícil concebir a los TP como "auténticas" enfermedades/trastornos mentales (Millon, 1981). De hecho, un aspecto importante del enfoque dimensional fue "su transformación de la psiquiatría tradicional (modelo biologista de Kraepelin) hacia un modelo de continuidad entre lo normal y lo anormal (pues la mayor parte de la gente puede tener algún grado de anormalidad en algún momento de la vida), mediante el cual la psiquiatría había pasado de ser una disciplina relacionada con la locura a una disciplina relacionada con la normalidad" (Sandín, 2013, p. 257).

En cualquier caso, también es importante tener en cuenta la alta tasa de comorbilidad entre los diversos TP, que es sobre todo fomentada por un sistema de clasificación categorial como lo es el DSM, ya que "la multiplicidad diagnóstica es un fenómeno iatrogénico ligado a todos los trastornos de la personalidad y un signo evidente de la dificultad para clasificar a estos pacientes" (Salavera, Puyuelo, Tricás y Lucha, 2010, p. 465). Aun empleando instrumentos de evaluación como el Inventario Clínico Multiaxial de Millon (MCMI), que en principio parte de un enfoque dimensional, hay que tener una extremada cautela. En efecto, se ha comprobado que el MCMI (al igual que otros autoinformes) parece presentar una tendencia al sobrediagnóstico (Fernández-Montalvo y Echeburúa, 2006) frente a pruebas como el International Personality Disorder Examination (IPDE), dando un alto índice de comorbilidad entre los diversos TP (Fernández-Montalvo, Landa, López-Goñi y Lorea, 2006). Esto puede determinar los resultados encontrados en diversos estudios realizados con poblaciones también diversas (por ejemplo, alcohólicos, adictos a la cocaína, personas sin hogar, etc).

En cuanto a la psicopatía, desde luego que se trata de "un constructo polémico, de gran relevancia clínica y criminológica" (Vinet, Alarcón y Pérez-Luco, 2011, p. 706). Así, los numerosos intentos de establecer una adecuada conceptualización parecen afirmar que no existe un acuerdo unánime ni respecto de la denominación, ni acerca del constructo o definición de psicopatía, puesto que ha sido definido desde diversos puntos de vista, transitando desde los puramente biológicos, hasta los más ambientalistas (López-Miguel y Núñez-Gaitán, 2009). En consonancia con la definición propuesta por Patrick (2006), la psicopatía es concebida como un síndrome, entendido como un conjunto de síntomas y no como un trastorno clínico. En este sentido, la consideración de la psicopatía como un tipo de psicopatología o trastorno mental es errónea, puesto que uno de sus criterios esenciales es la completa ausencia de cualquier tipo de manifestación psicopatológica (Cleckley, 1941). A tenor de lo anterior, no cabe considerar la psicopatía como un trastorno de la personalidad, definido por una serie de conductas y rasgos característicos que socialmente no se consideran positivos, en contraste con la conceptualización del propio Hare (2003b).

Atendiendo a las definiciones anteriormente expuestas, no podemos equiparar el término psicopatía al de trastorno de personalidad antisocial (TAP), al contrario de lo propuesto por numerosos autores (Cruz, Montiel y Aldana, 2014; Linares, 2007; Ramos et al., 2018). Concluimos que ambas entidades miden constructos diferentes, ya que mientras que el TAP se basa en conductas antisociales y delictivas, la psicopatía se centra en síntomas interpersonales y afectivos (Esbec y Echeburúa, 2014; López-Miguel y Núñez-Gaitán, 2009; Lorenzo, Agustina, Gómez-Durán y Martín-Fumadó, 2016; Torrubia y Cuquerella, 2008).

Por otro lado, comprobamos que este síndrome no está recogido en los manuales de clasificación y diagnóstico psicopatológico, atendiendo, principalmente, a cuestiones de política criminal relacionadas con la inimputabilidad, ya que las facultades intelectivas y volitivas de los psicópatas están totalmente intactas (Esbec y Echeburúa, 2010; Lorenzo et al., 2016; Pozueco, 2011). En este sentido, la psicopatía únicamente es tenida en cuenta a efectos de eximente -completa o incompleta-, cuando es acompañada de una enfermedad o trastorno mental (Esbec y Echeburúa, 2010).

Concluimos que la psicopatía no se circunscribe ni al ámbito delictivo ni al más general de las conductas antisociales (Pozueco, 2010), a diferencia de lo propuesto por López (2013), y mucho menos puede equipararse con cualquier tipo de psicopatología o trastorno mental (Cleckley, 1976; Pozueco, 2011). Por tanto, el perfil que aquí hemos esbozado sobre el psicópata responde bien al perfil subclínico y no delictivo trazado por Cleckley (1976), así como al perfil delictivo ampliado y modificado por Hare (2003) para sus estudios con internos penitenciarios, y ha sido corroborado por diversas investigaciones (Esbec y Echeburúa, 2010, 2014; López-Miguel y Núñez-Gaitán, 2009; Lorenzo et al., 2016; Torrubia y Cuquerella, 2008). Así, es evidente que la psicopatía no puede enfocarse como un trastorno, ni de la personalidad (aunque incluya rasgos de personalidad que también se han corroborado en la población general) (Drislane et al., 2014; Halty y Prieto, 2011; Savard, Brassard, Lussier y Sabourin, 2015; Seara, Neumann, Roiser, McCrory y Viding, 2013), ni de cualquier otra índole, fundamentalmente porque el calificativo de "trastorno" conlleva evidentes connotaciones psiquiátricas y/o psicopatológicas, y estas, por definición y si estableciéramos un diagnóstico diferencial de rigor, excluirían cualquier concepción de la psicopatía como una psicopatología o trastorno mental. En este sentido, las repercusiones derivadas de equiparar diagnósticos psicopatológicos con la psicopatía son evidentes, tal y como veníamos señalando, en el ámbito de la justicia criminal (Pozueco, 2011). 


\section{Referencias}

American Psychiatric Association (APA) (1994). The diagnostic and statistical manual of mental disorders (DSMIV) (4a ed.). Washington, DC: APA.

American Psychiatric Association (APA) (2013). Diagnostic and statistical manual of mental disorders (DSM-5) (5a edición). Washington, DC: APA.

Bateman, A., Fonagy, P. (2004). Psychotherapy for borderline personality disorder: Mentalization-based treatment. Oxford: Oxford University Press.

Belloch, A. (2010). Personalidad y trastorno de la personalidad. En A. Belloch y H. Fernández-Álvarez (Coords.), Tratado de trastornos de la personalidad (pp. 2146). Madrid: Síntesis.

Belloch, A., Fernández-Álvarez, H. (2002). Trastornos de la personalidad. Madrid: Síntesis.

Caballo, V., Salazar, I., e Irurtia, M. (2011). Trastornos de la personalidad: Características clínicas y diagnósticas. En V. Caballo, I. Salazar y J. Carrobles (Dirs.), Manual de psicopatología y trastornos psicológicos (2a ed.) (pp. 397-432). Madrid: Pirámide.

Clarkin, J. F. (2006). Conceptualization and treatment personality disorders. Psychotherapy Research, 16, 1-11.

Cleckley, H. M. (1941). The mask of sanity: An attempt to clarify the so-called psychopathic personality (1st Ed.). St. Louis, MO: Mosby.

Cleckley, H. M. (1976). The mask of sanity: An attempt to clarify the so-called psychopathic personality (5 ed.). St. Louis: Mosby.

Cruz, B., Montiel, D. y Aldana, G. (2014). Análisis de las conductas consideradas como parte del trastorno de personalidad antisocial: una mirada desde la teoría Gestalt. Revista Digital Universitaria, 15(1), 1-15.

Drislane, L., Patrick, C., Sourander, A., Sillanmäki, L., Aggen, S., Elonheimo, H., Parkkola, K., Multimäki, P., Kendler, K. S. (2014). Distinct variants of extreme psychopathic individuals in society at large: Evidence from a population-based sample. Personality Disorders: Theory, Research, and Treatment, 5(2), 154-163.

Echeburúa, E., Salaberría, K., Cruz, M. (2014). Aportaciones y limitaciones del DSM-5 desde la psicología clínica. Terapia Psicológica, 32(1), 65-74.

Esbec, E., Echeburúa, E. (2010). Violencia y trastornos de personalidad: implicaciones clínicas y forenses. Actas Españolas de Psiquiatría, 38(5), 249-261.

Esbec, E., Echeburúa, E. (2014). La evaluación de los trastornos de personalidad según el DSM-5: recursos y limitaciones. Terapia Psicológica, 32(3), 255-264. doi: 10.4067/S0718-48082014000300008

Eysenck, H. (1977). Crime and personality (3a ed.). London: Rotledge and Kegan.

Fariña, F., Vázquez, M. J., Arce, R. (2014). ¿Está mediada la gravedad delictiva y cronicidad de los delincuentes juveniles por la competencia cognitivo-comportamental? Universitas Psychologica, 13(3), 881-894.

Fernández-Montalvo, J., Echeburúa, E. (2006). Uso y abuso de los autoinformes en la evaluación de los trastornos de personalidad. Revista de Psicopatología y Psicología Clínica, 11(1), 1-12.

Fernández-Montalvo, J., Landa, N., López-Goñi, J. J., Lorea, I. (2006). Personality disorders in alcoholics: A comparative pilot study between the IPDE and the MCMI-II. Addictive Behaviors, 31(8), 1442-1448.
Garrido, V. J. (2000). El psicópata: Un camaleón en la sociedad actual. Alcira, Valencia: Algar.

Garrido, V. J. (2004). Cara a cara con el psicópata. Barcelona: Ariel.

Halty, L., Prieto, M. (2011). La psicopatía subclínica y la tríada oscura de la personalidad. Psicología Conductual, 19(2), 317-331.

Hare, R. (1970). Psychopathy: Theory and research. New York: Wiley.

Hare, R. (1982). Psychopathy and the personality dimensions of psychoticism, extraversion and neuroticism. Personality and Individual Differences, 3(1), 35-42.

Hare, R. (1993). Without conscience: The disturbing world of the psychopaths among us. New York: Pocket Books.

Hare, R. (2003a). The Hare Psychopathy Checklist-Revised (PCL-R) (2a ed.). Toronto: Multi-Health Systems.

Hare, R. (2003b). Sin conciencia: El inquietante mundo de los psicópatas que nos rodean. Barcelona: Ediciones Paidos.

Johns, J., Quay, H. C. (1962). The effect of social reward on verbal conditioning in psychopathic and neurotic military offenders. Journal of Consulting Psychology, 26(3), 217-220.

León, E., Zúñiga, D. P. (2012). Características psicopáticas en la adolescencia: Sistematización teórica. Universitas Psychologica, 11(4), 1197-1207.

Linares, J. L. (2007). La personalidad y sus trastornos desde una perspectiva sistémica. Clínica y Salud, 18(3), 381-399.

López, S. (2013). Revisión de la psicopatía: pasado, presente y futuro. Revista Puertorriqueña de Psicología, 24(2), 1-16.

López-Miguel, M. J. y Núñez-Gaitán, M. C. (2009). Psicopatía versus trastorno antisocial de la personalidad. Revista Española de Investigación Criminológica (REIC), 7(1), 1-17.

Lorenzo, F., Agustina, J., Gómez-Durán, E., Martin-Fumadó, C. (2016). Trastornos de la personalidad en la jurisprudencia española. Revista Española de Medicina Legal, 42(2), 62-66.

Lykken, D. T. (1995). The antisocial personalities. Hillsdale, NJ: LEA.

Malatesti, L. y McMillan, J. (Eds.) (2010). Responsibility and psychopathy: Interfacing law, psychiatry and philosophy. Oxford: Oxford University Press.

Marietán, H. R. (2011). No son psicópatas, pero lo parecen. ALCMEÓN, Revista Argentina de Clínica Neuropsiquiátrica, 17(1), 74-93.

McCord, W. M.. McCord, J. (1964). The psychopath: An essay on the criminal mind. New York: Van Nostrand.

Millon, T. (1981). Disorders of personality: DSM-III, Axis II. New York: Wiley.

Millon, T. (2011). Disorders of personality: Introducing a DSM/ ICD spectrum from normal to abnormal (3a ed.). New York: Wiley.

Millon, T., Davis, R. D. (1995). Disorders of personality: DSM-IV and beyond. New York: Guilford Press.

Millon, T., Davis, R. D. (1998). Ten subtypes of psychopathy. En T. Millon, Simonsen, E., Birket-Smith, M. y Davis, R. D. (Eds.), Psychopathy: Antisocial, criminal, and violent behavior (pp. 161-170). New York: The Guilford Press.

Millon, T., Grossman, S., Millon, C., Meagher, S., Ramnath, R. 
(2006). Trastornos de la personalidad en la vida moderna ( $2^{\mathrm{a}} \mathrm{ed}$.). Barcelona: Masson.

Millon, T., Simonsen, E., Birket-Smith, M. (1998). Historical conceptions of psychopathy in the United States and Europe. En T. Millon, E. Simonsen, M. Birket-Smith, R. Davis (Eds.), Psychopathy: Antisocial, criminal, and violent behavior (pp. 3-31). New York: Guilford Press.

Ortíz-Tallo, M., Cardenal, V., Ferragut, M. y Cerezo, M. V. (2011). Personalidad y síndromes clínicos: Un estudio con el MCMI-III basado en una muestra española. $R e$ vista de Psicopatología y Psicología Clínica, 16(1), 49-59.

Patrick, C. J. (2006). Back to the future: Cleckley as a guide to the next generation of psychopathy research. En C. J. Patrick (Ed.), Handbook of psychopathy (pp. 605-617). New York, NY: Guilford Press.

Partridge, G. (1930). Current conceptions of psychopathic personality. American Journal of Psychiatry, 10, 53-99.

Pelechano, V., De Miguel, A., Hernández, M. (2009). Trastornos de personalidad. En A. Belloch, B. Sandín y F. Ramos, Manual de psicopatología, Vol. I/ (Edición revisada) (pp. 449-480). Madrid: McGraw-Hill.

Pozueco, J. (2010). Psicópatas integrados: Perfil psicológico y personalidad. Madrid: EOS Psicología Jurídica.

Pozueco, J. (2011). Psicopatía, trastorno mental y crimen violento: Aspectos clínico-forenses, médico-legales y criminológicos. Madrid: EOS Psicología Jurídica.

Pozueco, J., Moreno, J. M., Blázquez, M., García-Baamonde, M. E. (2013). Psicópatas integrados/subclínicos en las relaciones de pareja: Perfil, maltratopsicológico y factores de riesgo. Papeles del Psicólogo, 34(1), 32-48.

Prado, J., García, J., Sánchez, S. (2013). Funcionamiento de la terapia cognitivo-interpersonal en los trastornos de la personalidad: Estudio de dos casos. Revista de Psicopatología y Psicología Clínica, 18(2), 139149.

Ramos, C., Duque-Grajales, J., Rendón, J., Montoya-Betancur, A., Baena, A., Pineda, D., Tobón, C. (2018). Cambios en el EEG en reposo de exparticipantes en el conflicto armado colombiano con trastorno de personalidad antisocial. Revista Colombiana de Psiquiatría, 47(2), 90-97. doi: 10.1016/j.rcp.2017.02.001

Salavera, C., Puyuelo, M., Tricás, J. M., Lucha, O. (2010). Comorbilidad de trastornos de personalidad: Estudio en personas sin hogar. Universitas Psychologica, 9(2), 457-467.

Sandín, B. (2013). DSM-5: ¿Cambio de paradigma en la clasificación de los trastornos mentales? Revista de Psicopatología y Psicología Clínica, 18(3), 255-286.

Savard, C., Brassard, A., Lussier, Y., Sabourin, S. (2015). Subclinical psychopathic traits and romantic attachment in community couples: A dyadic approach. Personality and Individual Differences, 72, 128-134.

Schneider, K. (1923). Die psychopathischen persönlichkeiten. Viena: Deuticke.

Schneider, K. (1980). Las personalidades psicopáticas (8ª ed.). Madrid: Morata.

Seara, A. B., Neumann, C. S., Roiser, J., McCrory, E., Viding, E. (2013).Investigating associations between empathy, morality and psychopathic personality traits in the general population. Personality and Individual Differences 52(1), 67-71.
Skeem, J. L., Cooke, D. J. (2010). Is criminal behavior a central component of psychopathy? Conceptual directions for resolving the debate. Psychological Assessment, 22(2), 433-445.

Torrubia R., Cuquerella A. (2008). Psicopatía: una entidad clínica controvertida pero necesaria en psiquiatría forense. Revista Española de Medicina Legal, 34, 25-35.

Vinet, E., Alarcón, P., Pérez-Luco, R. (2011). Detección y descripción de rasgos psicopáticos en adolescentes utilizando el MACl. Universitas Psychologica, 10(3), 705-719.

Wells, R. (1988). A fresh look at the muddy waters of psychopathy. Psychological Reports, 63(3), 843-856. 